\title{
The temperature dependence of magnetic susceptibility of solid oxygen
}

\author{
A. Jeżowski ${ }^{1}$, Z. Litwicki ${ }^{1}$, V.V. Sumarokov ${ }^{1,2}$, and P. Stachowiak ${ }^{1}$ \\ ${ }^{1}$ W. Trzebiatowski Institute of Low Temperature and Structure Research, \\ Polish Academy of Sciences 50-950 Wroclaw, Poland \\ E-mail: a.jezowski@int.pan.wroc.pl \\ ${ }^{2}$ B. Verkin Institute for Low Temperature Physics and Engineering \\ of the National Academy of Sciences of Ukraine, 47 Lenin Ave., Kharkov 61103, Ukraine \\ E-mail: sumarokov@ilt.kharkov.ua
}

Received July 10, 2006, revised August 9, 2006

\begin{abstract}
Temperature dependence of magnetic susceptibility of polycrystalline oxygen samples has been measured in the range from 1 to $54 \mathrm{~K}$. The measurements were performed using our home-designed SQUID-based gradiometer. The sensitivity of the instrument with respect to susceptibility reaches values of the order of $10^{-13}$. The results obtained are compared with earlier literature data.
\end{abstract}

PACS: 75.20-g, 75.20.Ck, 75.30.Cr, 75.50.Ee

Keywords: oxygen, magnetic susceptibility, phase transitions.

\section{Introduction}

Solid oxygen holds distinguished place among cryocrystals* as a substance exhibiting properties of both a magnet and a simple molecular crystal. The magnetic properties of crystalline oxygen originate from the fact that in the ground electron state an oxygen molecule has the electron spin equal 1. This unique combination of $\mathrm{O}_{2}$ properties stimulated its intensive investigation $[1,2]$.

Under saturated vapor pressure solid oxygen exists in three crystallographic phases. The low-temperature phase $\alpha$ has a monoclinic base-centered structure of $C 2 / m$ space group with ordered arrangement of the axes of molecules, and is magnetically-ordered. The phase $\beta$ has rhombohedral lattice of $R \overline{3} m$ space group and characterizes itself by strong short-ranged magnetic order. The phase $\gamma$ has the eight-molecule cubic elementary cell with orientationally-disordered structure of $P m 3 n$ space group and short-ranged quasi-one-dimensional magnetic order.

The pioneering absolute measurements of magnetic susceptibility of solid oxygen were done by Perrier and Kammerlingh-Onnes [3]. Later, both absolute and relative-method investigations of $\chi^{\mathrm{O}_{2}}$ were performed [3-9]. Usually, for relative measurements, the instruments were calibrated against the absolute data obtained in the liquid phase [2]. However, Meier et al. [8] have noticed that the results of measurements of $\chi$ in liquid oxygen can vary even by $10 \%$. Consequently, the calibration of an instrument against liquid $\mathrm{O}_{2}$ data can lead to appreciable systematic error. Despite almost hundred years history of $\chi^{\mathrm{O}_{2}}$ measurements, there is still considerable discrepancy in data obtained by various investigators, which may be due to various reasons, e.g., unreliable referencing to liquid $\mathrm{O}_{2}$ data, widely varying quality of samples, varying sample growth procedure, etc.

Until recently, the measurements of $\chi^{\mathrm{O}_{2}}$ were performed with standard techniques. Recently, commercial superconducting quantum interference devices (SQUIDs) become available, which feature the maximum sensitivity among any existing magnetic flux sensors [10]. SQUIDs can be used for measurement of various physical quantities, in particular for magnetic susceptibility. For the measurement of magnetic

* $\quad$ This term was firstly proposed by A.F. Prikhot'ko in 1976 
susceptibility $\chi$ of cryocrystals and their solid solutions, we designed and constructed [11] a facility equipped with a SQUID (made by «Quantum Design» company).

The purpose of this work was the measurement of temperature dependence of magnetic susceptibility of polycrystalline $\mathrm{O}_{2}$ samples in all crystal phases with aid of our SQUID gradiometer.

\section{Experimental}

Investigation of temperature dependence of magnetic susceptibility of solid oxygen in the temperature range $1-54 \mathrm{~K}$ was performed in the apparatus described elsewhere [11].

A characteristic feature of experimental investigation of cryocrystals is that growth of samples occurs directly in the measurement cell. Earlier we have elaborated methods of obtaining good quality oxygen samples [12]. Crystalline samples were grown by condensation of vapor on cold surface (reverse sublimation). (We note that in Refs. 3-9 the samples were obtained by crystallization from liquid phase.)

Our samples were grown and measured in a cylindrical ampoule, made of fused quartz of high purity. The ampoule was $60 \mathrm{~mm}$ high with $3 \mathrm{~mm}$ inner diameter, and $1 \mathrm{~mm}$ wall thickness. The temperature of condensation was slightly below the temperature of $\mathrm{O}_{2}$ triple point $(54.36 \mathrm{~K})$. The rate of crystal growth was about $6 \mathrm{~mm} / \mathrm{h}$. The height of sample was about a half of the height of the ampoule. The volume of the sample was $0.21 \mathrm{~cm}^{3}$, about an order less than commonly investigated $\mathrm{O}_{2}$ crystals. Although the measurement set-up does not allow crystal structure determination, our experience in crystal growth for measurement of thermal conductivity of solid oxygen [12] ensures that we are able to produce fine-grained polycrystalline samples.

The ampoule with the sample is mounted inside experimental chamber which in turn is placed inside a superconducting magnet, and can be moved along its axis.

For the susceptibility measurement, a 1st order gradiometer, connected to a SQUID, is employed. It consists of two coils with opposite turns direction, displaced to the distance equal the half of the ampoule height. Since the signal from the gradiometer equals the sum of signals from both coils (of opposite signs), the spurious signal from the ampoule material can be fully compensated. The SQUID magnetometer can detect minimal value of susceptibility of the order of $10^{-13}$. High sensitivity of the instrument allows measurement of small samples.

The system for preparation and analysis of gaseous samples was, in principle, similar to that described in
Refs. 12,13. It enables to compose samples, check their constituents with aid of a mass spectrometer and control the amount of gas(es) condensed into the ampoule.

Gaseous oxygen was obtained by thermal decomposition of $\mathrm{KMnO}_{4}$. Chemical purity was $99.99 \% \mathrm{O}_{2}$. Mass-spectrometer analysis of the samples show that the remaining $0.01 \%$ impurity was mainly $\mathrm{CO}_{2}$ which was easily frozen-out.

The processes of sample growth and measurement in the temperature region above liquid helium were accomplished by continuous flow of gaseous helium through measurement chamber, controlled by a system of thermometers, electric heaters and low-temperature expansion valve. For investigations below $4.2 \mathrm{~K}$, the measurement chamber was filled with liquid helium and its temperature was maintained by controlled pumping-out helium vapors with an automatic pressure regulator.

The temperature was controlled with DT-470 and TG-120-PL thermometers (Lake Shore Cryotronics) were used. The error of the sample temperature determination was below $0.1 \mathrm{~K}$.

The susceptibility measurements were conducted with both directions of temperature change. The rate of the sample temperature change was equal $0.5 \mathrm{~K} / \mathrm{min}$ outside the region of $\beta-\gamma$ transition. It is worth emphasizing that the occurrence of phase transitions (particularly the $\beta-\gamma$ transition which characterizes itself by large volume change) raises considerable problems with obtaining high-quality solid samples. Therefore, the phase transition regions were traversed slowly. The time of passing the $\beta-\gamma$ transition region was as long as $10 \mathrm{~h}$. We note that if the rate of passing the $\gamma-\beta$ transition was higher than $0.2 \mathrm{~K} / \mathrm{h}$, a hysteresis appeared.

\section{Discussion}

The absolute measurements of magnetic susceptibility of solid $\mathrm{O}_{2}$ were performed in works $[3,4,8]$. In the paper by Borovik-Romanov [4] only one absolute measurement point was taken in solid phase at the temperature $20.3 \mathrm{~K}$, the other points were relative measurements. The most complete absolute investigation was performed by Meier et al. [8]. Their paper reports two series of measurements, differing in the time of passing of $\gamma-\beta$ transition region. For one series (henceforth referred to as curve 1) this time was $2 \mathrm{~h}$, for the other (curve 2) $-0.5 \mathrm{~h}$.

Description of the experiments referred to in works [5,9] does not clarify which method, absolute or relative, was employed in the measurements.

Usually, during relative measurements, the results were linked to the data for liquid oxygen obtained by 
Kammerlingh-Onnes. However, from the work of Meier et al. [8] one knows that irreproducibility of the results of $\chi$ measurements can reach $10 \%$, hence instrument calibration against liquid oxygen values can lead to large systematic errors. In the high-temperature phase $\gamma$, the results depend strongly on the previous history of the sample. Upon crystallization of oxygen from liquid phase, one encounters a jump increase in magnetic susceptibility. Various authors report jumps of different height, changing from 0 to $13 \%$. In the low-temperature phase $\alpha, \chi$ is very sensitive to the sample anisotropy, whereas no such dependence is observable in $\beta-\mathrm{O}_{2}$. Therefore, our measurement system was calibrated against the data from absolute measurements of $\chi^{\mathrm{O}_{2}}$ [8] taken in the middle of the region of existence of $\beta$-phase (i.e. at about $34 \mathrm{~K}$ ), remote from phase transitions. The error of these results amounts $\sim 1 \%$.

Obtained in our experiments $\chi(T)$ values for solid $\mathrm{O}_{2}$ are shown in Fig. 1. In the $\alpha$-phase, raising of temperature results in weak increase of susceptibility, enhancing in the vicinity of $\alpha-\beta$ transition. At this transition, $\chi$ changes in a jump of about $35 \%$. The increase of susceptibility with temperature in $\beta$-phase is stronger than in $\alpha-\mathrm{O}_{2}$. The jump of $\chi$ at the $\beta-\gamma$ transition reaches the value of $55 \%$. In the phase $\gamma-\mathrm{O}_{2}$, increase of temperature causes decrease of $\chi$.

Comparison of our data with the data of Refs. [3-9] was presented in Fig. 2. First of all, note the great scatter of data coming from various authors. Differences in data for the investigated phases of solid oxygen reach values: about $33 \%$ for $\alpha-\mathrm{O}_{2}, 22 \%$ for $\beta-\mathrm{O}_{2}$ and $10 \%$ for $\gamma-\mathrm{O}_{2}$.

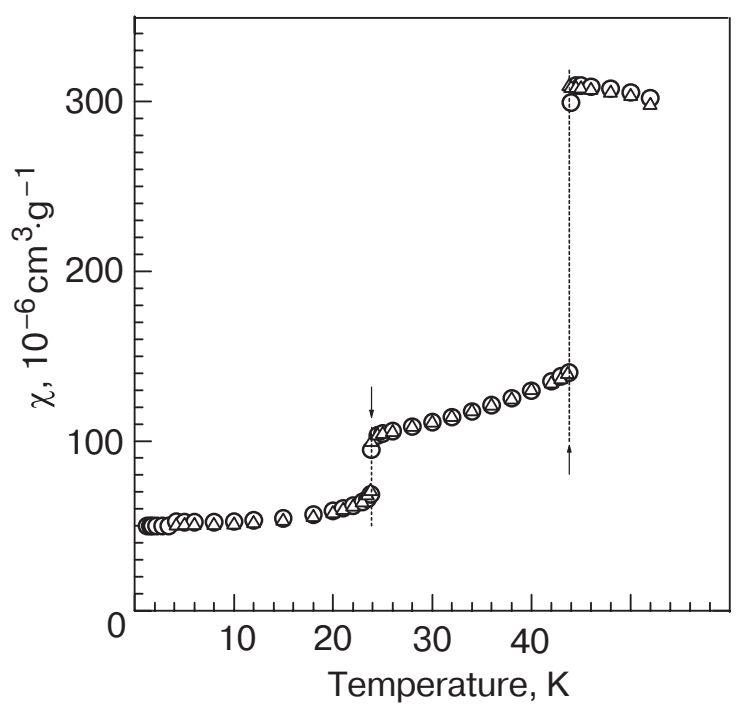

Fig. 1. Temperature dependence of magnetic susceptibility $\chi(T)$ of solid oxygen. Experimental data: 0 - raising temperature, $\triangle-$ lowering temperature.
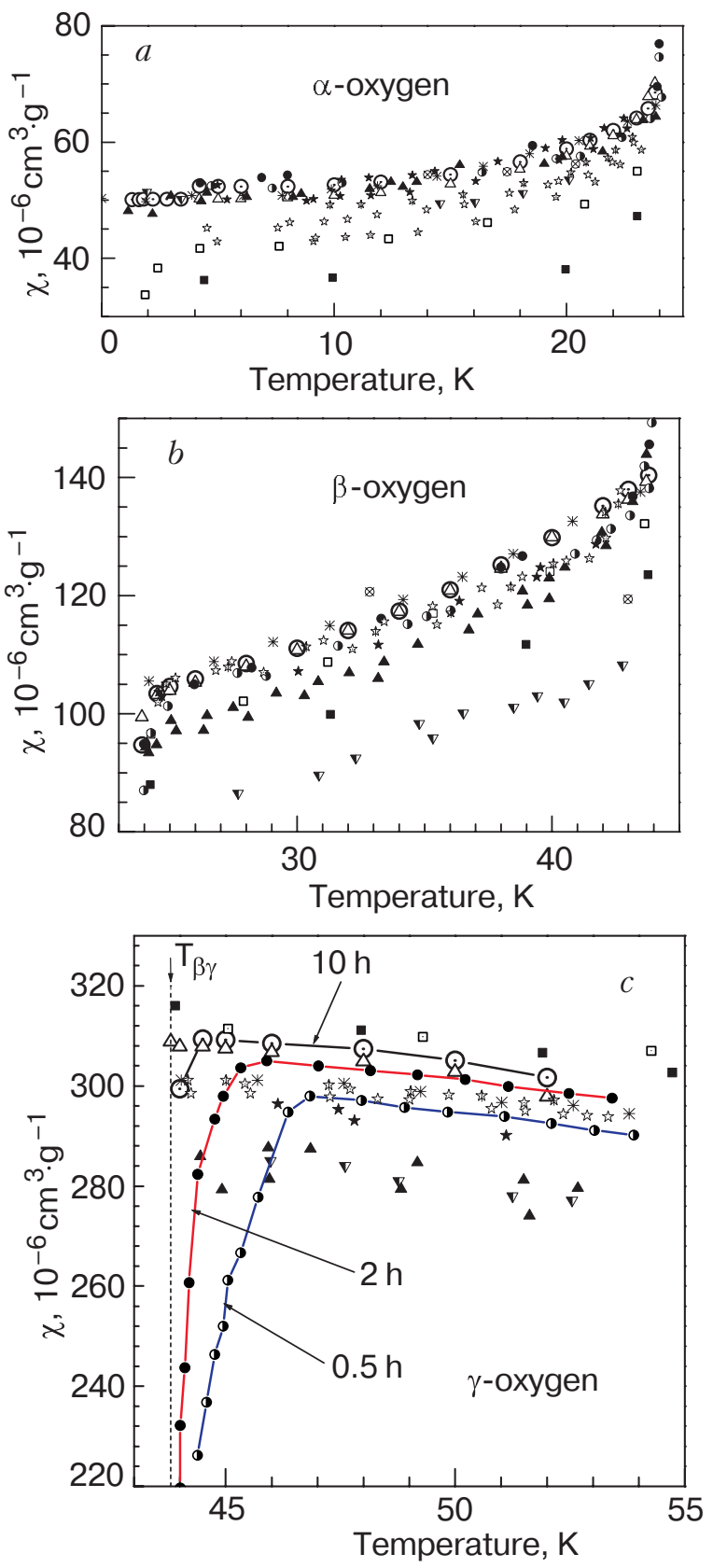

Fig. 2. Temperature dependence of $\chi$ for $\alpha-\mathrm{O}_{2}(a), \beta-\mathrm{O}_{2}(b)$, and $\gamma-\mathrm{O}_{2}(c)$. Comparison with experimental data from literature: $\odot, \triangle-$ the present work; $\otimes-$ Ref. 3; $\nabla-$ Ref. 4; $\square, \boldsymbol{\square}-$ Ref. 5 ; $\boldsymbol{\Delta}$ - Ref. 6; open-stars - polycrystalline samples with defferent predominating orientation and $\star-$ powder samples from Ref. 7; filled circles and half-filled circles - curves 1 and 2, respectively, from Ref. 8 ; $*-$ from Ref. 9.

As one can see in Fig. 2, $a$, the obtained by us experimental results for $\alpha-\mathrm{O}_{2}$ coincide with both absolute $[3,8]$ and relative [6] data from other measurements. In the work of DeFotis [7], there are reported data from two powder and three polycrystalline samples. Our results agree with his for powder samples, which also sustains the claim that our samples are fine-grained. The polycrystalline samples of the cited 
work [7] varied in that the dominating orientation was different; the $\chi(T)$ data varied, laying distinguishingly below our data. The results of the work [7] lay considerably below the data of all other works. The possible reasons for that variation could be difference in the predominating crystalline orientation as well as errors in relative data adjusting. The most recent data of Uyeda et al. [9] coincide with our results.

The results of our measurements of $\chi(T)$ in the $\beta$-phase $\mathrm{O}_{2}$ (Fig. 2,b) agree with the absolute data of Ref. 8, as well as with data of DeFotis [7] and Uyeda et al. [9], whereas the results of Refs. 4-6 are systematically lower.

The results of investigation of $\chi(T)$ in $\gamma-\mathrm{O}_{2}$ are depicted in Fig. 2,c. The curve 1 from [8] practically coincides with that obtained in our experiments, deviating in the vicinity of $\beta-\gamma$ transition, which is probably connected with the different time of passing that transition. The curve 2 from the cited paper runs lower, in particular in the vicinity of the transition (the time of passing $\beta-\gamma$ transition is four times shorter than for curve 1). The data of DeFotis [7] and of Uyeda [9] fall between our results and the curve 2 from Ref. 8 . Data of Kanda et al. [5] lay slightly above ours, whereas data from papers [4] and [6] fall considerably below ours. Comparing of the collected data shows that the time of passing the high-temperature transition has considerable influence on the results of $\chi$ of $\gamma-\mathrm{O}_{2}$ close to the $\beta-\gamma$ point.

\section{Conclusion}

We report that dense, fine-grained, polycrystalline samples of oxygen were obtained directly from gaseous phase, omitting liquid phase. $\chi(T)$ measurements on solid oxygen - made by relative method-in the 1-54 K range with aid of SQUID gradiometer were done for the first (known to us) time. Application of 1st order gradiometer allows to eliminate the contribution to susceptibility of the ampoule material. High sensitivity of the measurement set-up permits to investigate very small samples. Adjustment of the data was performed in the middle of the temperature region of $\beta$-phase existence according to the data from absolute measurements of Ref.8. The results obtained coincide with the absolute data of $\chi$ obtained in the paper [8] for all the three crystallographic phases, with data on powder samples [7] of $\alpha$-phase, and also with latest data [9]. Comparing the results to literature data points out to strong dependence of the values for $\gamma-\mathrm{O}_{2}$ in the vicinity of the $\beta-\gamma$ transition on the time of passing that transition.

1. Kriokristally, B.I. Verkin and A.F. Prikhot'ko (eds), Naukova Dumka, Kiev (1983) [in Russian].

2. Yu.A. Freiman and H.J. Jodl, Phys. Rept. 401, 1 (2004).

3. A. Perrier and H. Kamerlingh-Onnes, Commun. Leiden No 139, 25 (1914).

4. A.S. Borovik-Romanov, ZhETF 21, 1303 (1951); A.S. Borovik-Romanov, M.P. Orlova, and P.G. Strelkov, DAN SSSR 99, 699 (1954).

5. E. Kanda, T. Haseda, and A. Otsubo, Physica 20, 1316 (1954).

6. H.C. Jamieson and A.C. Hollis-Hallett, in: Proc. 10th Int. Conf. on Low Temperature Physics, M.P. Malkov (ed), VINITI, Moscow (1966), p. 158.

7. G.C. DeFotis, Phys. Rev. B23, 4714 (1981).

8. R.J. Meier, C.J. Schinkel, and A. de Visser, J. Phys. C15, 1015 (1982).

9. C. Uyeda, K. Sugiyama, and V. Date, J. Phys. Soc. Jpn. 54, 1107 (1985).

10. Dzh. Klark, Principy dejstvija $i$ primenenie SKVIDov, TIIER 77, 118 (1989) [in Russian]. Shortened version of: J. Clarke, Principles and Applications of SQUIDs, in: Superconducting Electronics, Proc. of NATO Advanced Study Institute] Il Ciocco, Lucca (Italy), (1988), H. Weinstock and M. Nissenoff (eds.), Springer-Verlag, Berlin (1989).

11. Z. Litwicki, A. Jeżowski, V.V. Sumarokov, and P. Stachowiak, Cryogenics (2006) submitted.

12. A. Jeżowski, P. Stachowiak, V.V. Sumarokov, J. Mucha, and Yu.A. Freiman, Phys. Rev. Lett. 71, 97 (1993).

13. A. Jeżowski and P. Stachowiak, Cryogenics 32, 601 (1992). 